Please do not remove this page

RMIT

UNIVERSITY

\title{
Lateral leakage in TM-like whispering gallery mode of thin-ridge silicon-on-insulator disk resonators
}

Nguyen, Giang Thach; Tummidi, Ravi; Koch, Thomas; Mitchell, Arnan

https://researchrepository.rmit.edu.au/esploro/outputs/9921864020901341/filesAndLinks?institution=61RMIT_INST\&index=null

Nguyen, G. T., Tummidi, R., Koch, T., \& Mitchell, A. (2009). Lateral leakage in TM-like whispering gallery mode of thin-ridge silicon-on-insulator disk resonators. Optics Letters, 34(7), 980-982.

https://doi.org/10.1364/OL.34.000980

Document Version: Published Version

Published Version: https://doi.org/10.1364/OL.34.000980

Repository homepage: https://researchrepository.rmit.edu.au

(C) 2009 Optical Society of America.

Downloaded On 2023/04/26 11:18:40 +1000

Please do not remove this page 


\title{
Lateral leakage in TM-like whispering gallery mode of thin-ridge silicon-on-insulator disk resonators
}

\author{
Thach G. Nguyen, ${ }^{1, *}$ Ravi S. Tummidi, ${ }^{2}$ Thomas L. Koch, ${ }^{2}$ and Arnan Mitchell ${ }^{1}$ \\ ${ }^{1}$ School of Electrical and Computer Engineering, RMIT University, GPO Box 2476V, Melbourne, VIC 3001, Australia \\ ${ }^{2}$ Center for Optical Technologies, Lehigh University, Bethlehem, Pennsylvania 18015, USA \\ *Corresponding author: thach.nguyen@rmit.edu.au
}

Received January 23, 2009; revised February 23, 2009; accepted February 24, 2009; posted February 25, 2009 (Doc. ID 106608); published March 20, 2009

\begin{abstract}
The leakage loss due to TM-TE mode coupling of TM-like whispering gallery mode in silicon-on-insulator (SOI) thin-ridge disk resonators is investigated for the first time to the best of our knowledge. We show that the propagation losses of TM-like mode in thin-ridge SOI disk resonators are significantly impacted by the radius of the disk. This behavior is predicted by a simple phenomenological model as well as a rigorous mode matching simulation. (C) 2009 Optical Society of America

OCIS codes: $130.2790,130.3120$.
\end{abstract}

Silicon photonic technology offers the potential for dense integration of photonic components while leveraging the benefits of the highly developed and mass manufacturable silicon platform. Typical silicon photonic waveguides are formed from thin films of silicon-on-insulator (SOI). The high index contrast between the silicon core and silica cladding leads to tightly confined modes. These offer the advantage of tight bends with low loss but can also limit interactions with the environment. Evanescent guiding may be desirable for optical sensors or hybrid photonic systems where nonlinearity or gain is provided by an external medium in close proximity to the silicon waveguide.

Thin shallow ridge waveguides [1] operating in the TM mode can exhibit highly evanescent fields but can also be subject to severe lateral radiation leakage losses even when perfectly smooth and straight [2]. We have recently demonstrated that these inherent losses can be effectively mitigated at particular "magic" widths where radiation from each boundary of the waveguide cancels coherently [3].

In addition to simple straight waveguides, practical integrated optic systems require more sophisticated structures including tapers, splits, couplers, and waveguide bends. Low-loss disk and ring resonators are also essential components for compact and efficient integrated optics. In this Letter we predict the lateral leakage for a thin-ridge SOI disk structure operating in TM polarization using rigorous mode matching. Strong dependence of the propagation loss on the resonator radius is predicted, and a new type of whispering gallery mode is identified.

Figure 1 presents the geometry of a thin-ridge SOI disk. The cross section and plan view are presented in Figs. 1(a) and 1(b), respectively. Consider a TM ray incident on the disk boundary. If the radius is large and the incident angle is glancing, the ray will be totally internally reflected. However, as with the straight waveguide counterpart [3], a small amount of power is coupled to transmitted and reflected TE polarized rays. The transmitted TE ray $\left(T_{\mathrm{TE}}\right)$ radi-

ates away from the origin, while the reflected TE ray $\left(R_{\mathrm{TE}}\right)$ traverses a secant across the disk and intersects the disk boundary a second time where it is largely transmitted. The totally internally reflected TM ray is guided along the disk boundary as a whispering gallery mode but continually looses power to the $\mathrm{TE}$ radiation.

The whispering gallery TM wave will meet the secant ray $\mathrm{TE}\left(R_{\mathrm{TE}}\right)$ at some point along the disk boundary and $R_{\mathrm{TE}}$ will interfere with $T_{\mathrm{TE}}$ generated at this point. Provided their relative phases are correct, the TE radiation may be canceled, resulting in low propagation loss. Through geometric reasoning the leakage minimum should occur for disks with radii that satisfy the resonant condition

$$
R=\frac{2 m+1+\left(\theta_{R}+\theta_{T}-\Delta \phi\right) / \pi}{4\left(\sqrt{\left(n_{\mathrm{TE}}^{\text {core }}\right)^{2}-N_{\mathrm{eff}}^{2}}-\theta_{R} \times N_{\mathrm{eff}}\right)} \lambda,
$$

where $m$ is an integer; $\theta_{R}=\arccos \left(N_{\text {eff }} / n_{\mathrm{TE}}^{\text {core }}\right)$ and $\theta_{T}$ $=\arccos \left(N_{\text {eff }} / n_{\mathrm{TE}}^{\text {clad }}\right)$ are the angles to the disk tangent of the reflected and transmitted TE rays, respectively; $n_{\mathrm{TE}}^{\text {core }}$ and $n_{\mathrm{TE}}^{\text {clad }}$ are the effective refractive indi-

(a)
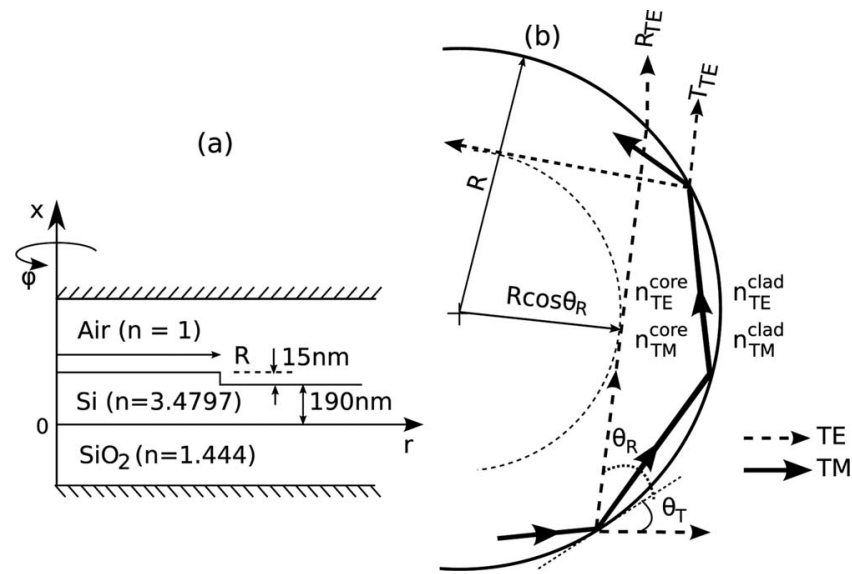

Fig. 1. (a) Cross section and (b) plan view and mode coupling diagram of a SOI thin-ridge disk resonator. 
ces of the TE slab modes in the disk and outside the disk, respectively; $N_{\text {eff }}$ is the effective index at the disk boundary of the TM-like whispering gallery mode; $\Delta \phi$ is the phase difference between the reflected and transmitted TE waves [4]; and $\lambda$ is the wavelength. Referring to Fig. 1, although the TE radiation outside the disk is resonantly canceled leading to low-loss propagation, there should be strong TE fields evident within the disk, extending from the disk boundary $(R)$ to a radius of $r=R \cos \theta_{R}$.

To rigorously model the wave propagation of TMlike modes in a thin-ridge SOI disk, we have employed a fully vectorial mode matching technique in cylindrical coordinates [5]. The disk was divided into radially uniform sections. The total field in each section was expressed as a superposition of the eigenmodes of the corresponding slab. The amplitudes of slab eigenmodes in each section are Bessel functions. The modes of the entire structure were then found by matching the field at the vertical interfaces between these sections. The eigenvalue solution is the complex azimuthal propagation constant with the imaginary component corresponding to the propagation loss, which includes both leakage and traditional bending losses. In the mode matching simulation, the computational window was open in the radial direction. Therefore, the leakage and radiation losses could be modeled very accurately.

As in [4], the continuum of radiation modes of each section was discretized by placing the structure between two perfectly conducting planes as shown in Fig. 1(a). The conducting planes do not affect the loss provided they are sufficiently far away from the Si core.

The mode matching method was used to calculate the azimuthal effective index $\left(N_{\text {eff }}\right)$, propagation loss and mode field profiles of the whispering gallery mode of the disk depicted in Fig. 1. The refractive indices, and geometric dimensions are also presented in Fig. 1. The Si core thickness and ridge height are identical to those of straight waveguides that were experimentally realized in [3]. The wavelength was set to $\lambda=1.55 \mu \mathrm{m}$. Simulations were conducted with nominal radii of $R=80,100,200$, and $400 \mu \mathrm{m}$ and were varied $\pm 2 \mu \mathrm{m}$ from these nominal values.

Figure 2 presents the propagation loss of the fundamental TM-like whispering gallery mode of the disk as a function of the disk radius as simulated us-

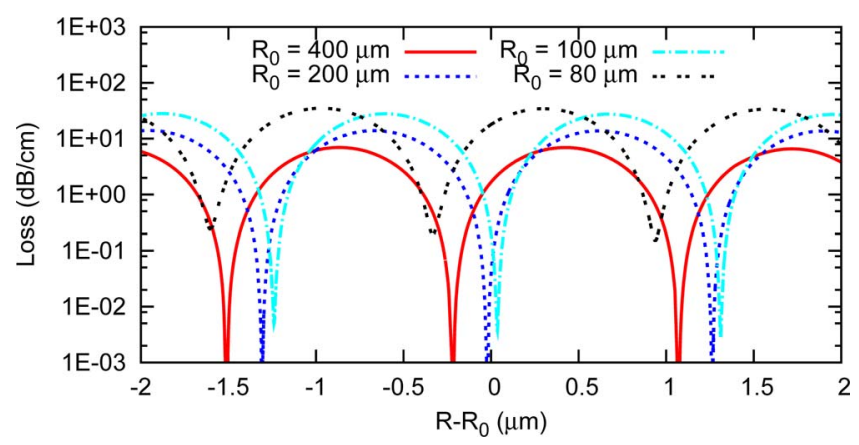

Fig. 2. (Color online) Loss of the fundamental TM-like whispering gallery mode of disk resonators as a function of the disk radius. ing mode matching. It can be seen that the propagation loss of the TM-like whispering gallery mode has a cyclic dependence on the disk radius. Low propagation loss is only possible at magic radii where the TE lateral leakage interferes destructively. The radius must be kept within $\pm 40 \mathrm{~nm}$ of the magic radius to ensure leakage loss less than $0.1 \mathrm{~dB} / \mathrm{cm}$. It is possible to improve the magic radius tolerance by implementing the technique proposed in [6]. Figure 2 shows that the locations of these magic radii, as predicted by mode matching, are in excellent agreement with the closed form geometric prediction of Eq. (1) where $R=199.98 \mu \mathrm{m}$ corresponds to $m=158$ th magic radius.

Comparing the maximum loss at $R_{0}=400,200,100$, and $80 \mu \mathrm{m}$, it is evident that the loss increases as the disk radius decreases. Traditional bending loss of a waveguide with equivalent effective refractive index contrast would account for only $0.17 \mathrm{~dB} / \mathrm{cm}$ at $R$ $=80 \mu \mathrm{m}$, however, the predicted increase in loss is far greater. With a smaller radius, the incident angle of the TM wave on the disk boundary will be greater. This larger angle results in greater TM-TE coupling [4] and hence greater loss.

When the disk radius is large, the traditional bending loss is negligible. However, for smaller disks $\left(R_{0}\right.$ $=80 \mu \mathrm{m})$, the traditional bending loss can make a significant contribution to the total loss, especially at the magic radii. As can be seen in Fig. 2, the propagation losses of disks with $R_{0}=80 \mu \mathrm{m}$ at magic radii do not approach zero, and instead are limited by the traditional bending loss of $0.17 \mathrm{~dB} / \mathrm{cm}$.

It should be noted that the simulated losses do not include the scattering loss due to surface roughness of the etched ridge. Using a thermal oxidation process [7], ultrasmooth waveguides have been practically realized providing very low scattering loss. It should be equally possible to fabricate disks with low scattering loss with this technique. For a shallow ridge structure, sidewall scattering will be less significant than for deep ridge counterparts. Further, it should be noted that the disk will support only a very few modes (five at $R_{0}=200 \mu \mathrm{m}$ ), and these are well separated in phase velocity, ensuring minimal coupling between modes.

Figures 3(a) and 3(b) shows the vectorial components of the electric field distributions of the fundamental TM-like whispering gallery modes of disk resonators with disk radii of $R=200.5 \mu \mathrm{m}$ (high loss) and $R=199.98 \mu \mathrm{m}$ (low loss), respectively. For both radii, the vertical field $\left(E_{x}\right)$ components show the typical distribution of the TM-like mode. Despite the mode being TM-like, there is a significant radial component $\left(E_{r}\right)$. When the disk is not at a magic radius, as shown in Fig. 3(a), a significant radially oriented field is evident in the region outside the disk corresponding to radiation loss. At the magic radius, as shown in Fig. 3(b), the radially directed field outside the disk is negligible. Inside the disk, the radial field is strong regardless of whether the mode is high or low loss. The component $E_{r}$ is strong for $r>125 \mu \mathrm{m}$, which is in excellent agreement with the prediction $r>\cos \theta_{R}$ using the ray model of Fig. 1 . The field is 

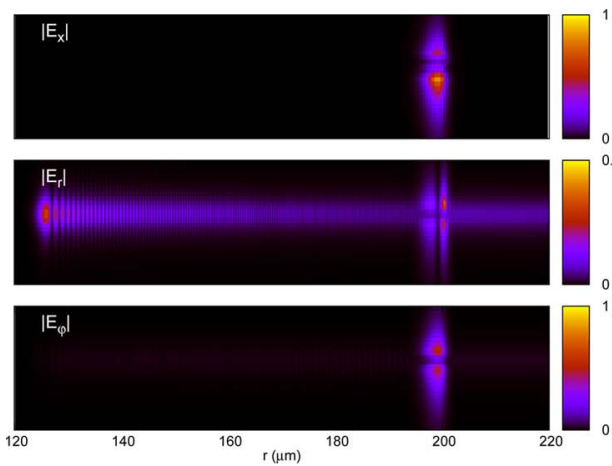

(a)
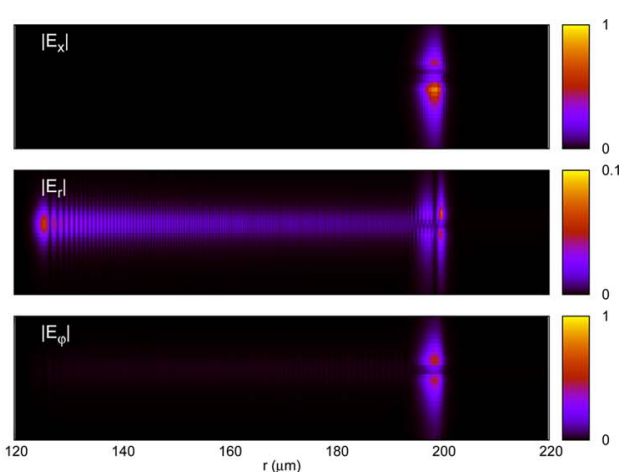

(b)

Fig. 3. (Color online) Electric field distributions of the fundamental TM-like whispering gallery mode for (a) 200.5 and (b) $199.98 \mu \mathrm{m}$ disks.

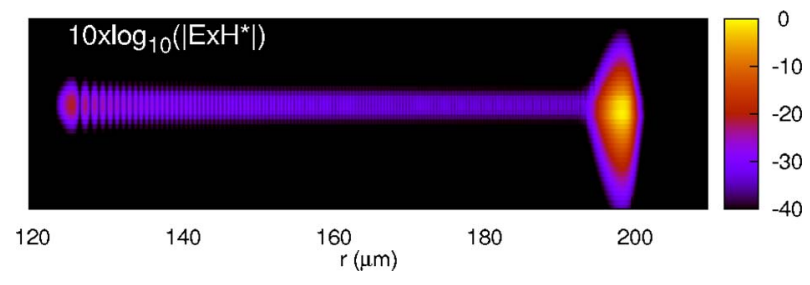

Fig. 4. (Color online) Power distribution of the fundamental TM-like whispering gallery mode of a disk with $R$ $=199.98 \mu \mathrm{m}$.

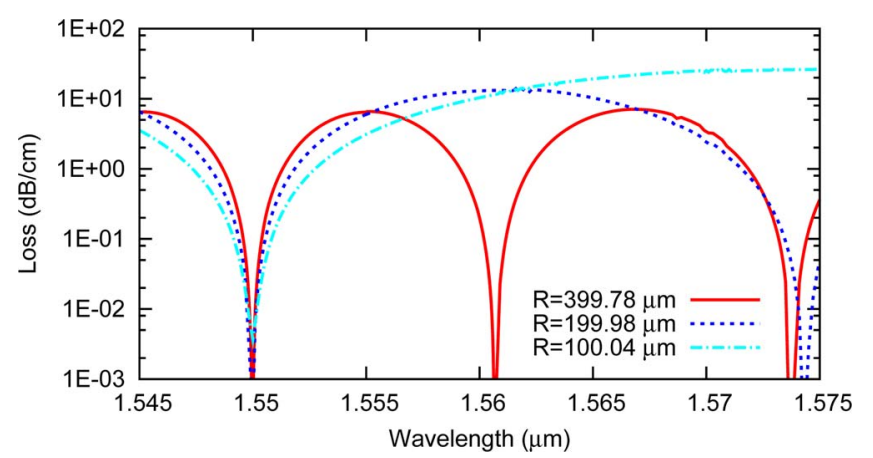

Fig. 5. (Color online) Loss of the fundamental TM-like whispering gallery mode as a function of wavelength.

strongest at $r=125 \mu \mathrm{m}$ and exhibits high contrast fringes close to this radius indicating that the secant rays are most coherent at this location.

Figure 4 presents the distribution of the Poynting vector amplitude of the fundamental whispering gallery TM-like mode of a disk with $R=199.98 \mu \mathrm{m}$ (low loss). It is perhaps surprising that a low loss whispering gallery mode can exist with optical power distributed over such a large cross-sectional area.

It is also worth noting that Eq. (1) predicts that the magic radius will be proportional to wavelength. Figure 5 presents the wavelength dependence of the propagation loss of disks with $R=399.78,199.98$, and $100.04 \mu \mathrm{m}$ which are magic radii at $\lambda=1.55 \mu \mathrm{m}$. The propagation loss of TM-like whispering gallery mode shows a cyclic dependence on the wavelength. Thus for a disk of given radius, light will resonate strongly only if the whispering gallery mode circumference is an integer number of wavelengths and if the wavelength and radius satisfies Eq. (1) to achieve low-loss propagation. This behavior offers potential for improved compact optical wavelength filtering.

In conclusion, we have presented the first analysis of the impact the TM-TE mode coupling on the leakage loss of cylindrically symmetric disks formed by SOI thin-ridge structure. A simple geometric model for prediction of the leakage behavior has been presented. Using the simple yet accurate phenomenological model and rigorous mode matching technique we have shown that the TM-like mode loss is strongly dependent on the disk radius. The existence of lowloss modes with broadly distributed cross section has been predicted. Wavelength-dependent propagation loss has also been predicted with possible application to high- $Q$ wavelength selective disk resonators.

\section{References}

1. M. A. Webster, R. M. Pafchek, G. Sukumaran, and T. L. Koch, Appl. Phys. Lett. 87, 231108 (2005).

2. K. Ogusu, J. Opt. Soc. Am. 73, 353 (1983).

3. M. Webster, R. Pafchek, A. Mitchell, and T. Koch, IEEE Photon. Technol. Lett. 19, 429 (2007).

4. T. G. Nguyen, R. S. Tummidi, T. L. Koch, and A. Mitchell, IEEE Photon. Technol. Lett. 10.1109/ LPT.2009.2013965 (2009).

5. L. Prkna, M. Hubálek, and J. Ctyroký, IEEE J. Sel. Top. Quantum Electron. 11, 217 (2005).

6. M. Koshiba, K. Kakihara, and K. Saitoh, Opt. Lett. 33, 2008 (2008).

7. R. Pafchek, R. Tummidi, J. Li, M. A. Webster, E. Chen, and T. L. Koch, Appl. Opt. 48, 958 (2009). 